\title{
Astrometric education in Saint-Petersburg State University, Russia
}

\author{
I. I. Kumkova and V. V. Vityazev \\ Sobolev Astronimical Instituute, Saint-Petersburg State University, 198504, Petrodvorets, \\ Universitetsky pr., 28., S. Petersburg, Russia \\ email: vityazev@list.ru
}

\begin{abstract}
The present system of astrometric education in the Saint-Petersburg State University is presented. The general courses, specific programs, seminars and observations that a student takes during a 5-year educational program are described.
\end{abstract}

Keywords. astrometry, education

\section{Introduction}

The Astronomy Chair at Saint-Petersburg University has existed since 1819. The names of the world wide known scientists, such as C.P. Glasenapp, A.A. Belopolsky, G.A. Tikhonov, V.V. Sharonov, K.F. Ogorodnikov, A.T. Agekyan, V.A. Ambartsumyan, V.V. Sobolev are connected with the University. The subject of special pride of the Astronomy Department is the fact that two former students who graduated from the Department, were later elected as presidents of International Astronomical Union (IAU). They are academicians V.A. Ambartsumian and A.A. Boyarchuck The current astronomical research in Saint-Petersburg University are focused on the following topics: fractal structure of the Universe, active galactic nuclei, spiral structure of our Galaxy, dark matter in galaxies, interaction of radiation and matter in different galactic objects, synthesis of elements in stars, stars with protoplanetary systems, radio emission of the Sun, dynamics of interplanetary matter, evolution of orbits in planetary and satellite systems, astronomical data analysis, stellar kinematics based on modern astrometric data, Earth Orientation Parameters from VLBI observations, and astrometric applications of the GPS/GLONASS techniques.

\section{Educational programme}

Annually, 20 students begin a 5-year program to become professional astronomers. During this time they study three principal sets of disciplines: (a) Mathematics, Numerical methods, Computer Science, Physics and Mechanics - 2000 h; (b) Astronomy - 2230 h; (c) Languages, History Philosophy, and others - $900 \mathrm{~h}$.

The first part of the astronomical set includes the following general courses: (a) General astronomy - 118 h; (b) Spherical astronomy - 72 h; (c) Celestial mechanics - 82 h; (d) Astrometry - 74 h; (e) General astrophysics - 104 h; (f) Theoretical astrophysics - 60 h; (g) Galactic astronomy - 56 h; (h) History of Astronomy - 42 h. The aim of general courses is to present the problems in various branches of modern astronomy. It is necessary to mention that the background of all subjects is a high level mathematics, programming and computer sciences, since the Astronomy Department is a part of Mathematics and Mechanics Faculty of the University. At the beginning of the 4-th year of 
study each student of the Astronomical Department should choose the specific area of the astronomy to continue his/her education. The offered areas are: Astrometry, Celestial Mechanics, Radio astronomy, Observational astrophysics, Theoretical astrophysics, and Galactic astronomy. The student also has additional courses connected with the chosen field of astronomy. For example, a student who is specializing in astrometry has special courses, such as: (a) VLBI astrometry - 32 h; (b) GPS/GLONASS astrometry - 32 h; (c) Space astrometry - 32 h; (d) Small field astrometry - 98 h; (e) The instruments of optical ground-based astrometry - 32 h; (f) Rotation of the Earth - 32 h; (g) Geodynamics - $64 \mathrm{~h}$; (h) Relativity in astrometry and Celestial mechanics - $32 \mathrm{~h}$. Each year, up to 5 students choose Astrometry, while the rest choose Astrophysics and Celestial mechanics where they have their own sets of special courses. The faculty of the Astronomical Department is in charge of providing the general astronomical courses, whereas the special courses are provided mainly by the leading scientists from Pulkovo Observatory and Institute of Applied Astronomy. $720 \mathrm{~h}$ of practical astrometric work is added to the theoretical courses mentioned above since research is an indispensable part of the graduate program. For example, students in astrometry have opportunities to work with faculty and staff of Pulkovo Observatory and of Institute of Applied Astronomy on a broad range of astrometric problems. At the end of the 5-year educational program, a student presents the results of a research project that is evaluated by independent scientists. If the student is successful, the student is awarded the first degree - Specialist corresponding to a Master of Science degree in the USA. The second degree, Candidate of Science, (the counterpart of $\mathrm{PhD}$ ) is awarded to a person after completing a Postgraduate course (up to 3 years) and presenting a thesis based on the results of his/her scientific activity. The third and highest degree is Doctor of Science, which is awarded for large contribution to science.

\section{Conclusion}

Where can a former graduate student with a degree from Saint-Petersburg University find a job? There are a few institutions in Saint-Petersburg interested in hiring astrometrists, such as Pulkovo observatory of RAS, the Institute of Applied Astronomy of RAS, and several institutions connected with space geodesy research. If this is not successful, then our astrometrists may go into computer science or industry where the acquired math and programming skills are highly valued. 